\title{
Stellar rotation at young ages: new results from Corot's monitoring NGC 2264
}

\author{
F. Favata ${ }^{1}$, G. Micela ${ }^{2}$, S. Alencar ${ }^{3}$, S. Aigrain ${ }^{4}$ and K. Zwintz ${ }^{5}$ \\ ${ }^{1} \mathrm{ESA}$ \\ ${ }^{2}$ Università di Palermo \\ ${ }^{3}$ Departmento de Física, ICEx, UFMG, Belo Horizonte, MG 31270-901, Brazil \\ ${ }^{4}$ University of Exeter \\ ${ }^{5}$ Institute for Astronomy, University of Vienna, Austria
}

Stellar rotation at young ages: new results from Corot's Angular momentum is one of the driving forces in the early evolution of stars. Issues such as the coupling between the star and the accretion disk (the so-called disk regulation paradigm), are traced by the evolution of rotational momentum, but affect the star-forming process as a whole. One of the features observed in star-forming regions (e.g. ONC and NGC 2264) of age between 1 and few Myr, for masses above 0.25 solar masses, is a bimodality of the rotational period distribution, with a peak around 1 day and the other at around 4 to 7 days. This bimodality has been interpreted as the smoking gun of the disk-locking mechanism (with the fast rotators having lost their disk and the slow ones still being regulated by their disks).

We recently observed the NGC 2264 star-forming region (age $3 \mathrm{Myr}$ ) with the Corot space telescope, obtaining an uninterrupted 24 day long high accuracy photometric series. This allows the determination of rotational periods for $\mathrm{P}<12$ days with high accuracy and the removal of any biases due to the coverage imposed by ground-based observational campaigns. The Corot photometric campaign on NGC 2264 unambiguously shows that stars with $\mathrm{M}>0.25 \mathrm{Msun}$ have a single-peaked distribution (with a peak at 3-4 days) and no short period peak. The bimodal distribution that has been reported based on groundbased campaigns is not present in the Corot-based data, and the short-period peak in the distribution (centered around 1 day) appears to be spurious and due to aliasing effects between the actual rotational modulation and the observational window function.

We will present the Corot-based rotational period data for NGC 2264 and discuss the implications of the shift from a double-peaked to a single-peaked distribution on the angular momentum evolution in the few Myr age range. In particular we will discuss the implications for the disk-locking paradigm, making also use of the available Spitzer data regarding the presence of disks in NGC 2264. 